\title{
Article \\ Optomechanically Induced Transparency and Slow-Fast Light Effect in Hybrid Cavity Optomechanical Systems
}

\author{
Qinghong Liao ${ }^{1,2, *}$, Weida Bao ${ }^{1}$, Xing Xiao ${ }^{1}$, Wenjie $\mathrm{Nie}^{3}$ and Yongchun Liu ${ }^{2, *(D)}$ \\ 1 Department of Electronic Information Engineering, Nanchang University, Nanchang 330031, China; \\ baowd123@sina.com (W.B.); xx23wy@sina.cn (X.X.) \\ 2 State Key Laboratory of Low-Dimensional Quantum Physics, Department of Physics, Tsinghua University, \\ Beijing 100084, China \\ 3 Department of Applied Physics, East China Jiaotong University, Nanchang 330013, China; \\ niewenjiezhu@sina.cn \\ * Correspondence: qhliao@ncu.edu.cn (Q.L.); ycliu@tsinghua.edu.cn (Y.L.)
}

Citation: Liao, Q.; Bao, W.; Xiao, X.; Nie, W.; Liu, Y. Optomechanically Induced Transparency and Slow-Fast Light Effect in Hybrid Cavity Optomechanical Systems. Crystals 2021, 11, 698. https://doi.org/ $10.3390 /$ cryst11060698

Academic Editors: Yuri Kivshar and Dmitri Donetski

Received: 26 April 2021

Accepted: 11 June 2021

Published: 18 June 2021

Publisher's Note: MDPI stays neutra with regard to jurisdictional claims in published maps and institutional affiliations.

Copyright: (c) 2021 by the authors. Licensee MDPI, Basel, Switzerland. This article is an open access article distributed under the terms and conditions of the Creative Commons Attribution (CC BY) license (https:// creativecommons.org/licenses/by/ $4.0 /)$.
Abstract: We theoretically investigate the optomechanically induced transparency (OMIT) phenomenon and the fast and slow light effects of a four-mode optomechanical system with the Kerr medium. The optomechanical system is composed of an array of three single-mode cavities and a mechanical oscillator. The three cavities are a passive cavity, a no-loss-gain cavity and a gain optical cavity, respectively. A Kerr medium is inserted in the passive cavity. We study the influence of the Kerr medium on the stability of the optomechanical system, and find that the stable regime of the optomechanical system can be adjusted by changing the Kerr coefficient. We demonstrate that the phenomenon of optomechanically induced transparency will appear when the Kerr medium exists in the optomechanical system and find that the frequency position of the absorption peak on the left increases linearly with the Kerr coefficient. In addition, we also investigate the fast and slow light effects in this system. The results show that we can control the fast and slow light by adjusting the Kerr coefficient, tunneling strength, and driving field strength. This study has potential application prospects in the fields of quantum optical devices and quantum information processing.

Keywords: Kerr medium; optomechanically induced transparency (OMIT); slow-fast light

\section{Introduction}

Over the last decade, cavity optomechanical systems, which explore the interaction between the optical cavity and mechanical mode, have received extensive attention in many rapidly developing fields [1], such as the cooling of mechanic modes to ground-state [2-5], quantum entanglement [6,7], photon blockade [8-15], mass sensing [16-18], parity-time (PT) symmetry [19-24], optical field control [25-29], etc. As a typical example of controlling the output spectrum of the optical field, optomechanically induced transparency (OMIT) is widely used in optical storage [30] and quantum information and communication processes [31,32]. The physical mechanism of OMIT is similar to electromagnetically induced transparency (EIT), which is essentially caused by the interference between the anti-Stokes scattering field and the probe field. In detail, when pump light and weak probe light act on the cavity at the same time, the anti-Stokes photon formed will interfere with the photon in the probe field, so that the absorption of the probe field by the optical cavity is zero. The interesting phenomenon was first proposed by Agarwal et al. [33,34], and verified by Weis et al. [35] and Painter et al. [36] in the experiment. Additionally, we can focus on the advantages of different quantum systems in the same hybrid cavity optomechanical system by the interaction of a mechanical resonator and an optical cavity. Therefore, the multiple-OMIT phenomena have been studied theoretically in hybrid cavity optomechanical systems [37-43], such as the triple optomechanical system [37], the L-G 
rotational-cavity optomechanical system [38], the atomic media-assisted optomechanical system [39-42], the microtoroid cavity optomechanical system [43], etc.

In addition, the adjustment of the output spectrum will cause rapid phase dispersion, which will change the group delay of the light pulses. We can achieve slow light or fast light effects by controlling the group delay of the output light field [44-47]. Generally, the probe field in a transparent window usually has rapid phase dispersion. This means that we can connect the multiple-OMIT phenomenon with the slow and fast light effect of the optomechanical system. The slow and fast light effect of the optomechanical system has attracted extensive attention in optical communication and interferometry [48,49], and the appearance of multiple-OMIT provides an important platform for further study of slow and fast effect. In recent years, many people have begun to study fast and slow light effects in different cavity optomechanical systems. Wang et al. [50] studied the slow or fast light effect in PT-symmetric mechanical systems and found that the amplification of group delay could be effectively controlled by the mechanical exceptional point. This provides a new way to adjust the switch from slow light to fast light. Additionally, Han et al. [41] theoretically studied the optical properties in an atom-assisted optomechanical system with a mechanical pump and demonstrated that the phase and amplitude of the external pump can be used to tune fast and slow light. Additionally, Ziauddin et al. [51] considered two atoms inside the optomechanical system to study slow and fast light propagation. Liu et al. discussed the nonreciprocal transmission and fast and slow light effects in the microtoroid cavity optomechanical system in Ref. [43].

Meanwhile, we know that the nonlinear optical effect can be significantly enhanced by adding the nonlinear medium into the optomechanical system. Therefore, the optomechanical system with the nonlinear medium has been widely investigated in recent years [52]. The effects of the nonlinear medium on the cooling, steady-state, and entanglement of optomechanical systems have been studied in Refs. [53-55]. In addition, there is a potential application in the field of the control of output optical spectra in the optomechanical system. Li et al. [56] added a degenerate optical parametric amplifier (OPA) and a higherorder-excitation atom into the optomechanical system, and theoretically studied the optical response of the output field and the fast and slow light. We can know from Ref. [57] that the existence of the Kerr medium can adjust the spectral position of OMIT.

Motivated by these developments, we consider a triple optomechanical system with the Kerr medium driven by the strong driving field and weak probe field, as shown in Figure 1. In the hybrid cavity optomechanical system, we study the effect of nonlinear enhancement in the passive cavity on the stability of the system. Additionally, we theoretically investigate the OMIT phenomenon and tunable fast and slow light in the hybrid cavity optomechanical system. The position of the absorption peak can be changed linearly by adjusting the Kerr coefficient, which provides a new way to measure the Kerr medium. In addition, we can control the fast and slow light phenomena by adjusting the Kerr coefficient, tunneling strength, and driving strength. Therefore, we provide a new method for the study of fast and slow light effects.
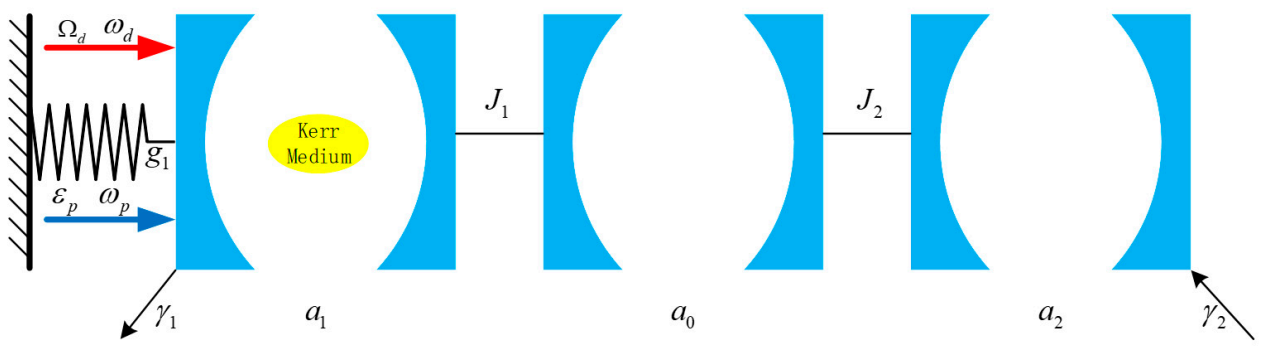

Figure 1. Schematic diagram of a hybrid cavity optomechanical system. It consists of three singlemode cavity arrays, a Kerr medium, and a mechanical oscillator. In addition, the passive cavity is driven by an external laser field and a weak probe field. 
The structure of the paper is as follows. In Section 2, the theoretical model of the optomechanical system is introduced and the linearized Hamiltonian is derived. In Section 3, we obtain the Heisenberg-Langevin equation of the system. Moreover, we obtain the Jacobian matrix and calculate the Lyapunov exponent by the equation. Additionally, we obtain the output field of the probe frequency. Then, we analyze the OMIT phenomenon in Section 4. Meanwhile, we discuss the fast and slow light effects of the system in Section 5. Finally, the conclusions are given in Section 6.

\section{Model}

As shown in Figure 1, we consider the hybrid cavity optomechanical system consisting of the optical trimer system containing a Kerr medium and a mechanical oscillator. The optical trimer system comprises a passive cavity $a_{1}$, a no-loss-gain cavity $a_{0}$ and an active cavity $a_{2}$, and the photon-tunneling strengths between two adjacent cavities are $J_{1}$ and $J_{2}$, respectively. We can change the tunneling strength between adjacent cavities by adjusting the distance between them. Meanwhile, the Kerr medium is inserted in the passive cavity with the Kerr coefficient of $\chi$. Here, the loss rate and the resonance frequency of the passive cavity $a_{1}$ are, respectively, $\gamma_{1}$ and $\omega_{c 1} ; \gamma_{2}$ and $\omega_{c 2}$ are the gain rate and the resonance frequency of active cavity $a_{2}$; and the resonance frequency of no-loss-gain cavity $a_{0}$ is $\omega_{c 0}$. In addition, the passive cavity $a_{1}$ interacts with the mechanical oscillator, and the optomechanical coupling strength is $g_{1}$. The decay rate and its resonance frequency of the mechanical oscillator are $\gamma_{m}$ and $\omega_{m}$, respectively. In addition, the optomechanical cavity is driven by a strong driving field $\Omega_{d}$ with frequency $\omega_{d}$ and a weak probe field $\varepsilon_{p}$ with frequency $\omega_{p}$. The total Hamiltonian of the hybrid cavity optomechanical system in the rotating frame at the frequency $\omega_{d}$ can be written as the following form:

$$
\begin{aligned}
H & =\Delta_{c 1} a_{1}^{+} a_{1}+\Delta_{c 0} a_{0}^{+} a_{0}+\Delta_{c 2} a_{2}^{+} a_{2}+\omega_{m} b^{+} b+\chi a_{1}^{+2} a_{1}^{2} \\
& -g_{1} a_{1}^{+} a_{1}\left(b^{+}+b\right)+J_{1}\left(a_{0}^{+} a_{1}+a_{0} a_{1}^{+}\right)+J_{2}\left(a_{0}^{+} a_{2}+a_{0} a_{2}^{+}\right) \\
& +i\left(a_{1}^{+} \Omega_{d}-a_{1} \Omega_{d}^{*}\right)+i\left(a_{1}^{+} \varepsilon_{p} e^{-i \delta t}-a_{1} \varepsilon_{p}^{*} e^{i \delta t}\right)
\end{aligned}
$$

where $\Delta_{c i}=\omega_{c i}-\omega_{d}(i=0,1,2)$ are the detunings of the three cavity modes from the driving field; $\delta=\omega_{p}-\omega_{d}$ is the detuning between the probe field and the pump field; and $\Omega_{d}=\sqrt{2 \kappa_{a} p_{d} / h \omega_{d}}$ and $\varepsilon_{p}=\sqrt{2 \kappa_{a} p_{p} / h \omega_{p}}$ are the amplitude of the strong driving field and the weak probe field, respectively. Here, $p_{d}\left(p_{p}\right)$ are the powers of the driving (probe) field. We can know from Equation (1) that the first three terms denote the free Hamiltonian of three single-mode cavities. Here, the operators $a_{1}\left(a_{1}^{+}\right), a_{0}\left(a_{0}^{+}\right)$and $a_{2}\left(a_{2}^{+}\right)$ represent the annihilation (creation) operators of photons in the passive cavity, no-loss-gain cavity, and gain cavity, respectively. The fourth term of the equation is the free Hamiltonian representing the mechanical oscillator, and the operator $b\left(b^{+}\right)$represents the annihilation (creation) operator of phonons. The next term represents the Hamiltonian of the Kerr medium. The next term represents the optomechanical coupling between the passive cavity and mechanical oscillator. The next two terms denote the interaction Hamiltonian of adjacent cavities. The last two terms of Equation (1) represent the interaction between the passive cavity and two input fields, i.e., strong pump field and weak probe field.

\section{Quantum Dynamics and Fluctuations}

For the convenience of discussing the dynamics of the hybrid cavity optomechanical system, we define the dimensionless position operator $\hat{x}=\left(b+b^{+}\right) / \sqrt{2}$ and momentum operator $\hat{p}=i\left(b^{+}-b\right) / \sqrt{2}$ instead of the phonon operator in Equation (1). Then, we can 
obtain the nonlinear dynamic equation of the optomechanical system by the quantum Heisenberg-Langevin equation, as shown:

$$
\begin{aligned}
& \dot{x}=\omega_{m} p \quad \dot{p}=-\frac{\gamma_{m} p}{2}-\omega_{m} x+\sqrt{2} g_{1} a_{1}^{+} a_{1}+\xi(t) \\
& \dot{a}_{1}=\left(-i \Delta_{c 1}-\frac{\gamma_{1}}{2}\right) a_{1}+i \sqrt{2} g_{1} a_{1} x-2 i x a_{1}^{+} a_{1}^{2}-i J_{1} a_{0}+\Omega_{d}+\varepsilon_{p} e^{-i \delta t}+\sqrt{2 \gamma_{1}} a_{i n 1}(t) \\
& \dot{a}_{2}=\left(-i \Delta_{c 2}+\frac{\gamma_{2}}{2}\right) a_{2}-i J_{2} a_{0}+\sqrt{2 \gamma_{2}} a_{i n 2}(t) \\
& \dot{a}_{0}=-i \Delta_{c 0} a_{0}-i J_{1} a_{1}-i J_{2} a_{2}
\end{aligned}
$$

where $a_{i n 1}(t), a_{i n 2}(t)$ and $a_{i n 3}(t)$ are the input vacuum noise operators in the cavity and satisfy the following relations $\left\langle a_{i n 1}(t)\right\rangle=\left\langle a_{i n 2}(t)\right\rangle=\left\langle a_{i n 3}(t)\right\rangle=0$ [58]. Additionally, $\xi(t)$ is quantum Brownian stochastic noise with zero mean value and satisfies the following relations $\left\langle\xi(t) \xi\left(t^{\prime}\right)\right\rangle=\frac{\gamma_{m}}{\omega_{m}} \int \frac{d \omega}{2 \pi} e^{-i \omega\left(t-t^{\prime}\right)} \omega\left(1+\operatorname{coth} \frac{h \omega}{2 k_{B} T}\right)$ [59]. Meanwhile, the dimensionless position operator and momentum operator of the mechanical oscillator satisfy the following relations $[x, p]=i$. Considering the interference from the probe field, we can decompose each operator as the sum of steady-state and the sum of fluctuations, i.e., $O=O_{s}+\delta O\left(O=x, p, a_{i=1,2,3}\right)$ [60]. We can further regard them as the sum of real and imaginary parts. Then, we can expand Equation (2) in this way. In the process of expansion, we can ignore the higher-order term and the probe field term $\varepsilon_{p} e^{-i \delta t}$. Thus, we can obtain the linearized dynamics of the system as follows: $f(t)=M f(t)$. The Jacobian matrix $M$ contains the stable or randomness characteristic of the system, and $f^{T}(t)=\left(\delta x(t), \delta p(t), \delta \operatorname{Re}\left[a_{1}(t)\right], \delta \operatorname{Im}\left[a_{1}(t)\right], \delta \operatorname{Re}\left[a_{2}(t)\right], \delta \operatorname{Im}\left[a_{2}(t)\right], \delta \operatorname{Re}\left[a_{0}(t)\right], \delta \operatorname{Im}\left[a_{0}(t)\right]\right)$ represent the column vector of the fluctuation operator. We can obtain the Jacobian matrix $M$ as follows:

$$
\left.\begin{array}{cccccc}
0 & 0 & 0 & 0 & 0 & 0 \\
2 \sqrt{2} g_{1} \operatorname{Re}\left[a_{1 s}\right] & 2 \sqrt{2} g_{1} \operatorname{Im}\left[a_{1 s}\right] & 0 & 0 & 0 & 0 \\
-\frac{\gamma_{1}}{2}+2 \chi \operatorname{Im}\left[a_{1 s}^{2}\right] & \Delta_{c 1}-\sqrt{2} g_{1} x_{s}+4 \chi\left|a_{1 s}\right|^{2}-2 \chi \operatorname{Re}\left[a_{1 s}^{2}\right] & 0 & 0 & 0 & J_{1} \\
-\Delta_{c 1}+\sqrt{2} g_{1} x_{s}-4 \chi\left|a_{1 s}\right|^{2}-2 \chi \operatorname{Re}\left[a_{1 s}^{2}\right] & -\frac{\gamma_{1}}{2}-2 \chi \operatorname{Im}\left[a_{1 s}^{2}\right] & 0 & 0 & -J_{1} & 0 \\
0 & 0 & \frac{\gamma_{2}}{2} & \Delta_{c 2} & 0 & J_{2} \\
0 & 0 & -\Delta_{c 2} & \frac{\gamma_{2}}{2} & -J_{2} & 0 \\
-J_{1} & J_{1} & 0 & J_{2} & 0 & \Delta_{c 0} \\
0 & 0 & -J_{2} & 0 & -\Delta_{c 0} & 0
\end{array}\right)
$$

We can see that there are two parameters $x_{s}$ and $a_{1 s}$ in the Jacobian matrix $M$. They represent the steady-state mean values of the mechanical oscillator and the passive cavity field, respectively. We can set the first derivative of operators $x$ and $a_{1}$ in Equation (2) to zero, and obtain the steady-state mean $x_{s}$ and $a_{1 s}$. Meanwhile, we need to assume that $a_{1 s} \gg 1$, i.e., there are a large number of photons in the passive cavity.

We can obtain the Lyapunov exponent of the system by finding the eigenvalues of the Jacobian matrix $M$ [61]. The Lyapunov exponent can reflect the stability of the nonlinear dynamic system. We know that when the Lyapunov exponent is positive, the system is unstable. Therefore, when all the Lyapunov exponents of the nonlinear dynamic system are negative, the system will be in a stable state, i.e., the maximum Lyapunov exponent is negative [62]. We can judge whether the optomechanical system is stable or unstable by calculating the maximum Lyapunov.

Then, we discuss the absorption of the probe field by the cavity. We can expand Equation (2) as follows [20,37,63]:

$$
\begin{aligned}
& O=O_{s}+{ }^{+} O \varepsilon_{p} e^{-i \delta t}+{ }^{-} O \varepsilon_{p}^{*} e^{i \delta t} \\
& \dot{O}=-i \delta^{+} O \varepsilon_{p} e^{-i \delta t}+i \delta^{-} O \varepsilon_{p}^{*} e^{i \delta t}
\end{aligned}
$$

where $O \in\left\{x, p, a_{1}, a_{0}, a_{2}\right\}$. To calculate the absorption of a weak probe field by cavity mode more conveniently, we ignore the higher-order term containing $\varepsilon_{p}$ under the condition of $O_{s} \gg O_{ \pm}$and probe field amplitude $\varepsilon_{p}$. At the same time, by substituting Equation (4) into 
Equation (2), and comparing the coefficients of both sides, we can obtain the steady-state solution and output field of the system as follows:

$$
x_{s}=\frac{\sqrt{2} g_{1}\left|a_{1 s}\right|^{2}}{\omega_{m}}, a_{1 s}=\frac{\Omega_{d}}{i \Delta+\frac{\gamma_{1}}{2}-\frac{J_{1}^{2}\left(-i \Delta_{c 2}+\frac{\gamma_{2}}{2}\right)}{J_{2}^{2}-i \Delta_{c 0}\left(-i \Delta_{c 2}+\frac{\gamma_{2}}{2}\right)}}
$$

and

$$
{ }^{+} a_{1}=\frac{\lambda_{5} \lambda_{7}}{-2 i \beta_{1} \chi \lambda_{6} a_{1 s}^{+}-4 \chi^{2}\left|a_{1 s}\right|^{4} \lambda_{7}+\lambda_{5} \lambda_{7} \lambda_{8}-\beta_{1} \lambda_{5} \lambda_{6}}
$$

where the undefined variables are $\Delta=\Delta_{1}+2 \chi\left|a_{1 s}\right|^{2}, \beta_{1}=i \sqrt{2} g_{1} \omega_{m}^{2} x_{s}=2 i g_{1}^{2} \omega_{m}\left|a_{1 s}\right|^{2}$, $\lambda_{1}=\frac{J_{2}^{2}}{i\left(\Delta_{c 2}-\delta\right)-\frac{\gamma_{2}}{2}}, \quad \lambda_{2}=\frac{J_{1}^{2}}{\left(\Delta_{c 0}-\delta\right)-i \lambda_{1}}, \quad \lambda_{3}=\frac{J_{2}^{2}}{-i\left(\Delta_{c 2}+\delta\right)-\frac{\gamma_{2}}{2}}, \quad \lambda_{4}=\frac{J_{1}^{2}}{\left(\Delta_{c 0}+\delta\right)+i \lambda_{3}}$, $\lambda_{5}=-i\left(\Delta_{1}+\delta+4 \chi\left|a_{1 s}\right|^{2}-\lambda_{4}\right)+\frac{\gamma_{1}}{2}, \lambda_{6}=\frac{2 i \chi\left|a_{1 s}\right|^{2}}{\lambda_{5}}+1, \lambda_{7}=\omega_{m}^{2}-\delta^{2}-\frac{i \gamma_{m} \delta}{2}+\frac{\beta_{1}}{\lambda_{5}}$, $\lambda_{8}=i\left(\Delta_{1}-\delta-4 \chi\left|a_{1 s}\right|^{2}-\lambda_{2}\right)+\frac{\gamma_{1}}{2}$.

Further, according to the input-output relationship of cavity $a_{1}: \varepsilon_{\text {out }}(t)+\varepsilon_{p} e^{-i \delta t}+$ $\Omega_{d}=\gamma_{1} a_{1}$, and then expanding $\varepsilon_{\text {out }}$ and $a_{1}$, comparing the coefficients of both sides, we can obtain ${ }^{+} \varepsilon_{\text {out }}=\gamma_{1}{ }^{+} a_{1}-1$. To study the response of the system to the weak probe field, we can write the amplitude of the rescaled output field to the weak probe field in the following form: $\varepsilon_{T}={ }^{+} \varepsilon_{\text {out }}+1=\gamma_{1}{ }^{+} a_{1}$ [37]. We can study the absorption and dispersion of the probe field by studying the real and imaginary parts of $\varepsilon_{T}$.

\section{The Multi-Transparency Windows of the Output Field for the Hybrid Optomechanical System}

Generally, the OMIT and fast-slow light phenomena are studied when the optomechanical system is in a stable state. If the system is asymptotically stable and keeps a long evolution time, the OMIT phenomenon can be obtained [42]. In Figure 2, we have observed the border between the stable and unstable regimes. Accordingly, the external parameters can be selected to satisfy the stability condition of the system. Therefore, the stability of the system should be studied in detail. We discuss the image of the maximum Lyapunov exponent as a function of the tunneling strength $J_{2} / \gamma_{1}$ and the gain-loss ratio $\gamma_{2} / \gamma_{1}$ with different Kerr coefficients in Figure 2. The passive cavity with decay rate $\gamma_{1}=2 \pi \times 215 \mathrm{kHz}$ is selected. The frequency of the mechanical oscillator is $\omega_{m}=23.4 \gamma_{1}$ and the damping rate $\gamma_{m}=2 \pi \times 141 \mathrm{~Hz}$. In addition, the detuning between the driving field and the cavity fields is $\Delta_{i}=\omega_{m}(\mathrm{i}=0,1,2)$, and the driving field amplitude $\Omega_{d}=10^{5} \gamma_{1}$. The tunneling strength between the passive cavity and no-loss-gain cavity is $J_{1}=0.3 \gamma_{1}$. In addition, we choose the optomechanical coupling strength $g_{1}=2 \times 10^{-5} \gamma_{1}$ [32,37]. When the value is taken in the regime below the line, the system is in a stable state. Otherwise, the system is in an unstable state. This curve stands for the boundary line between stable and unstable regimes. When the gain-loss ratio $\frac{\gamma_{2}}{\gamma_{1}}$ is small, the left edge of the image will not change significantly with the different Kerr coefficient $\chi$ in Figure 2. Similarly, for a fixed gain-loss ratio $\frac{\gamma_{2}}{\gamma_{1}}$, the corresponding tunneling strength $J_{2}$ has a maximum value of $J_{2}^{\max }$ and a minimum value of $J_{2}^{\min }$. The difference between the two values $\Delta J_{2}=J_{2}^{\max 2_{2}^{\min }}$ represents the width of the stable region corresponding to a given gain-loss ratio. It can be seen from Figure 2 that when the Kerr medium exists in the passive cavity, increasing the gain-loss ratio $\frac{\gamma_{2}}{\gamma_{1}}$ will reduce the width of the stable regime of the system. Meanwhile, for a given gain-loss ratio $\frac{\gamma_{2}}{\gamma_{1}}$, the width of the stable regime of the system will decrease with the increase in the Kerr coefficient $\chi$. Therefore, we can change the stable state of the system by controlling the Kerr medium strength. Additionally, when the Kerr medium strength is changed, the maximum gain-loss ratio corresponding to the tunneling strength $\frac{J_{2}}{\gamma_{1}}$ is almost constant. 


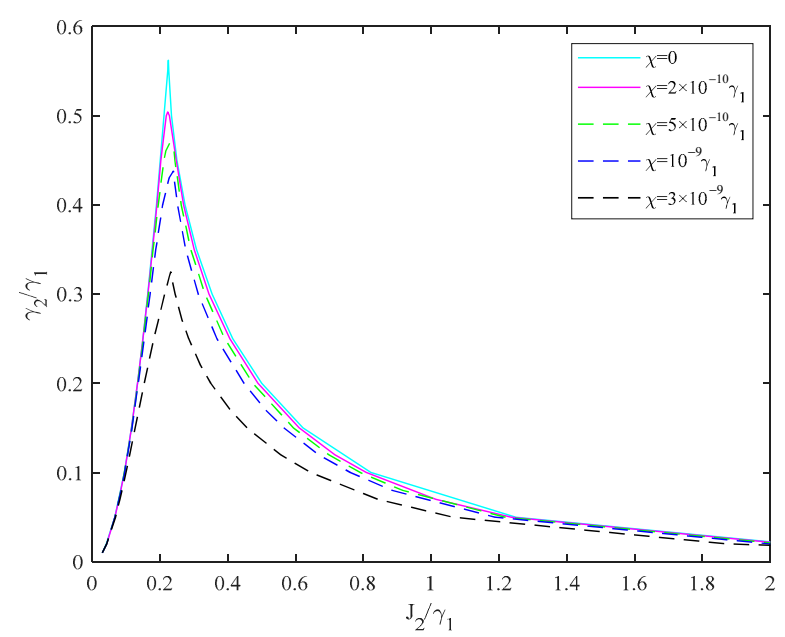

Figure 2. The image of the maximum Lyapunov exponent as a function of the tunneling strength $\frac{J_{2}}{\gamma_{1}}$ and the gain-loss ratio $\frac{\gamma_{2}}{\gamma_{1}}$ with different Kerr coefficient $\chi$. The parameters are $\gamma_{1}=2 \pi \times 215 \mathrm{kHz}$, $\omega_{m}=23.4 \gamma_{1}, \gamma_{m}=2 \pi \times 141 \mathrm{~Hz}, \Delta_{c i}=\omega_{m}(\mathrm{i}=0,1,2), \Omega_{d}=10^{5} \gamma_{1}, g_{1}=2 \times 10^{-5} \gamma_{1}, J_{1}=0.3 \gamma_{1}$.

In Figure 3, we plot the real part $(\chi=0)$ and $(\chi \neq 0)$ of the output filed as a function of the probe detuning $\frac{\delta}{\omega_{m}}$ with different $\gamma_{2}$. It can be seen from Figure $3 \mathrm{a}-\mathrm{c}$ that when the system is in a stable regime, there always exist two dips on the two sides of $\delta=\omega_{m}$. When the Kerr medium exists in the cavity optomechanical system, adjusting the value of $\gamma_{2}$ can make the system gradually transition from a stable regime to an unstable regime. We can see the absorption peaks on both sides move to the left after adding the Kerr medium in the process. Meanwhile, the width of the absorption peak on the left side increases slightly, and the width of the absorption peak on the right side decreases slightly. Moreover, we can see that the middle absorption peak is in a stable regime. With the increase in $\gamma_{2}$, the absorption rate of the hybrid cavity optomechanical system at $\delta=\omega_{m}$ begins to increase rapidly. Therefore, the strong resonance absorption appears in the optomechanical system, which is induced by the active cavity. In the presence of the Kerr medium, we can see from Figure $3 \mathrm{a}-\mathrm{c}$ that the absorption rate of the optomechanical system at $\delta=\omega_{m}$ increases faster than without the Kerr medium. In addition, the optomechanical system with the Kerr medium shows a dip at $\delta=\omega_{m}$, and the wave trough drops to a negative value, which means that the probe field is amplified significantly [64].
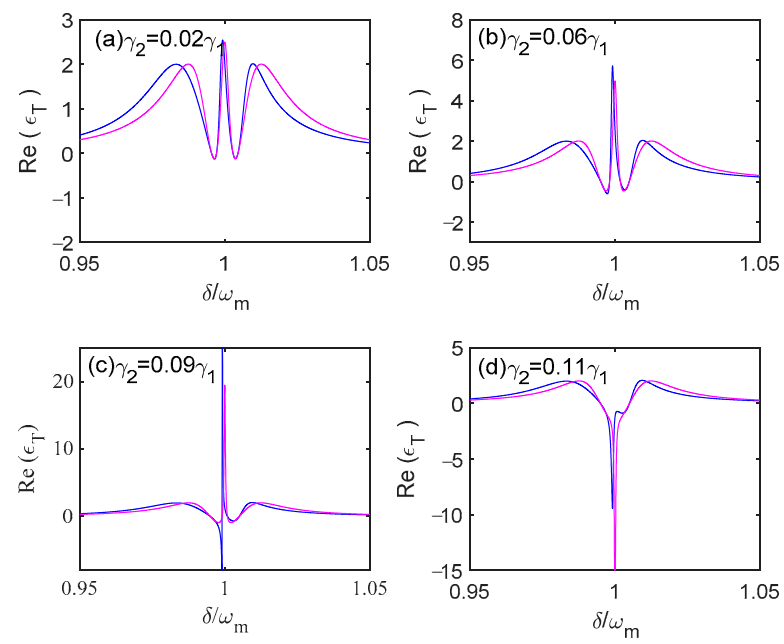

Figure 3. The real part $\left(\chi=3 \times 10^{-9} \gamma_{1}\right.$, blue line) and the real part ( $\chi=0$, pink line) of the output filed as a function of the probe detuning $\frac{\delta}{\omega_{m}}$ with different $\gamma_{2}$. (a) $\gamma_{2}=0.02 \gamma_{1}$; (b) $\gamma_{2}=0.06 \gamma_{1}$; (c) $\gamma_{2}=0.09 \gamma_{1} ;$ (d) $\gamma_{2}=0.11 \gamma_{1}$. $\left(g_{1}=0, J_{2}=0.095 \gamma_{1}\right.$, and the other parameter values are the same as in Figure 2). 
Then, we discuss the influence of Kerr medium strength on the system when the hybrid cavity optomechanical system in a stable regime. When the Kerr medium does not exist in the passive cavity, i.e., $\chi=0$, we can see that there are three absorption peaks in the blue solid curve in Figure 4a. When the Kerr coefficient of $\chi=5 \times 10^{-9} \gamma_{1}$, we can see that all three absorption peaks in Figure 4a start to move to the left. It can be seen from Figure $4 \mathrm{a}$ that the absorption peak on the left moves obviously compared with the absorption peak on the other sides. Further, when we increase the Kerr coefficient $\chi$, we can see that the width of the absorption peak on the left increases. In contrast, the width of the middle and right absorption peaks decreases with the increase in the Kerr coefficient $\chi$, and the decrease rate of the right absorption peak is slightly greater than the middle absorption peak. The reason is that the Kerr medium is added into the passive cavity, which makes the center frequency of the cavity field change, and the nonlinearity of the cavity field is enhanced. In addition, we can also find from Figure 4a that increasing the Kerr coefficient $\chi$ will increase the height of the absorption peaks in the middle and right. Meanwhile, the absorption peak in the middle increases more obviously. Therefore, the absorption rate of the absorption peak in the middle can be controlled by changing the Kerr coefficient. Next, we plot the position of the absorption peak on the left from $\delta=\omega_{m}$ as a function of the Kerr coefficient $\chi$ in Figure $4 \mathrm{~b}$. It is obvious that the position of the absorption peak from $\delta=\omega_{m}$ depends linearly on the Kerr coefficient. Therefore, we can easily adjust the position of the left absorption peak by changing the Kerr coefficient of $\chi$, which provides a new way to measure the Kerr medium.
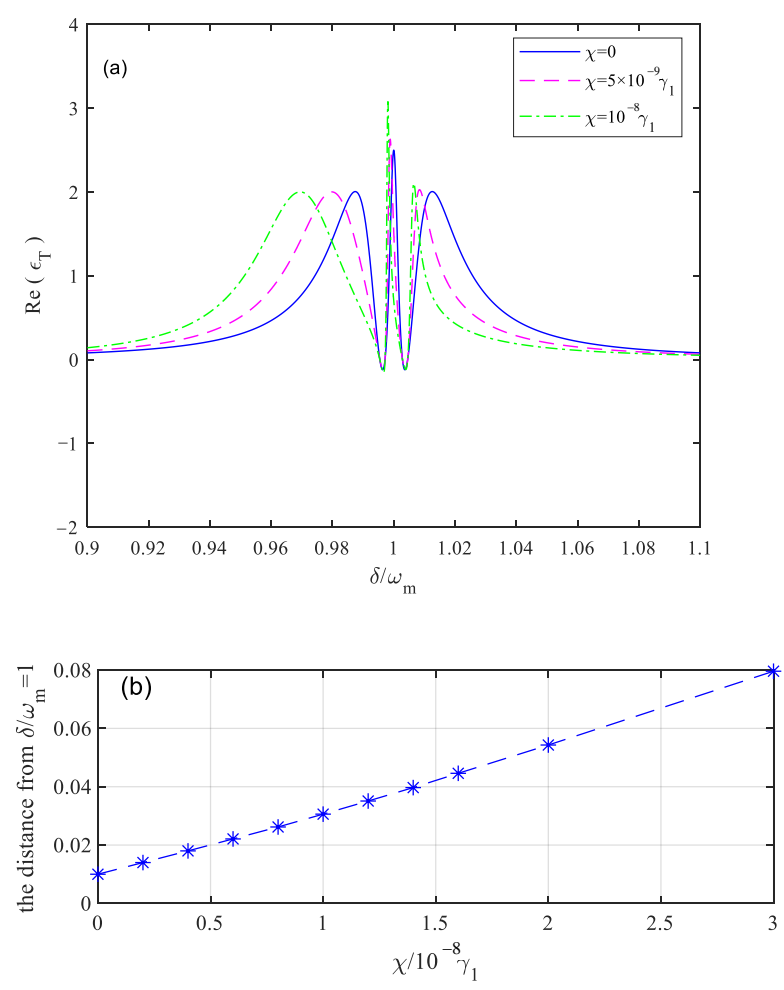

Figure 4. (a) The real part of the output field as a function of the probe detuning $\delta-\omega_{m}$ for different Kerr coefficient $\chi, \chi=0$ (black, solid curve); $\chi=5 \times 10^{-9} \gamma_{1}$ (pink, dashed curve); $\chi=10^{-8} \gamma_{1}$ (green, dash-dotted curve); (b) The distance from $\frac{\delta}{\omega_{m}=1}$ as a function of the different Kerr coefficient $\chi \cdot \gamma_{2}=0.02 \gamma_{1}$, and the other parameter values are the same as in Figure 3. 


\section{Slow-Fast Light Effect of the Output Field}

We theoretically investigate the slow-fast light phenomena in the optomechanical system. It is well known that the change of parameters in the system will cause rapid abnormal phase dispersion in the probe field. If the phase dispersion is positive, the light will propagate slowly. Similarly, if the phase dispersion is negative, the fast light phenomenon will appear. To study the group delay corresponding to the weak probe field, we use the following formula $[42,44]$ :

$$
\tau=\frac{\partial \phi}{\partial \omega_{p}}=\operatorname{Im}\left[\frac{1}{\varepsilon_{T}} \frac{\partial \varepsilon_{T}}{\partial \omega_{p}}\right]=\operatorname{Im}\left[\frac{1}{\varepsilon_{T}} \frac{\partial \varepsilon_{T}}{\partial \delta}\right]
$$

where $\phi=\arg \left(1-\varepsilon_{T}\right)$ is referred to as the phase of the transmission field and $\tau$ represents the group delay. $\tau>0$ and $\tau<0$ represent the slow light phenomenon and fast light phenomenon in the hybrid cavity optomechanical system, respectively.

First, we can discuss the influence of the change in the Kerr coefficient on the group delay. We can see that the amplitude of group delay is positive near $\delta=0.996 \omega_{m}$ and $\delta=1.004 \omega_{m}$ in Figure 5. At the same time, when we increase the Kerr coefficient, we can see that the peaks of the left and right peaks decrease. This shows that the group delay decreases near $\delta=0.996 \omega_{m}$ and $\delta=1.004 \omega_{m}$, which means that the slow light phenomenon in the system is weakened. Therefore, we can change the group delay by adjusting the Kerr coefficient in the passive cavity, thus changing the slow light phenomenon. When $\delta=\omega_{m}$, we can see that the group delay of the system is negative, which shows that there is a fast light phenomenon in the optomechanical system. When we increase the value of the Kerr coefficient $\chi$, the depth of the dip at $\delta=\omega_{m}$ begins to decrease. The phenomenon indicates that the fast light effect will decrease with the increase in the Kerr coefficient in the optomechanical system. It shows that we can change the fast light phenomenon by adjusting the Kerr coefficient $\chi$. It is because the emergence of the Kerr medium changes the center frequency of the passive cavity field, which makes the nonlinearity in the cavity enhanced [65]. The change in Kerr medium strength can significantly affect the slow-fast light phenomenon.

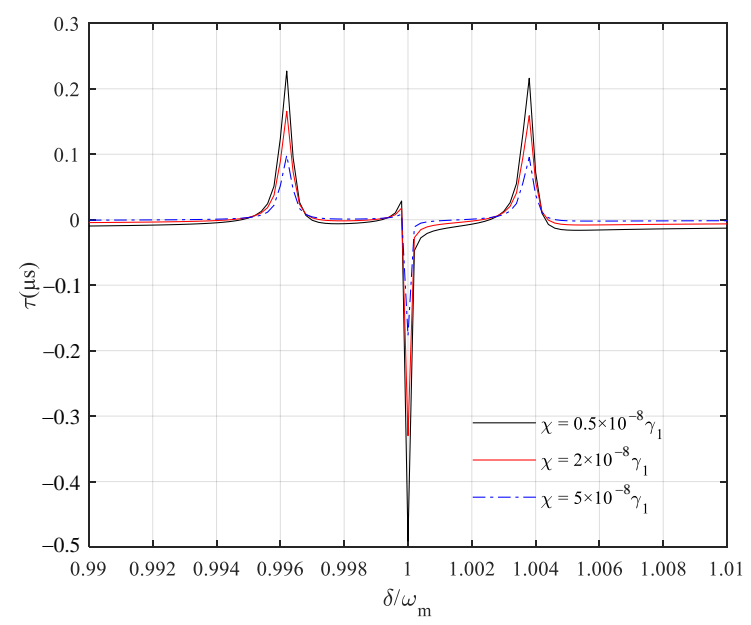

Figure 5. Group delay $\tau$ as a function of the probe detuning $\delta / \omega_{m}$ for different values of the Kerr coefficient $\chi \cdot \chi=5 \times 10^{-9} \gamma_{1}$ (black, solid curve); $\chi=2 \times 10^{-8} \gamma_{1}$ (red, dashed curve); $\chi=5 \times 10^{-8} \gamma_{1}$ (blue, dash-dotted curve). $g_{1}=2 \times 10^{-5} \gamma_{1}$, and the other parameter values are the same as in Figure 3.

Then, we can see from Figure 6 that the group delay of the output field is positive near the points $\delta=0.996 \omega_{m}$ and $\delta=1.004 \omega_{m}$, which corresponds to the slow light phenomenon of the hybrid optomechanical system. At the same time, when we increase the tunneling intensity between the passive cavity and no-loss-gain cavity, we can see that the peak 
values near $\delta=0.996 \omega_{m}$ and $\delta=1.004 \omega_{m}$ decrease, the group delay decreases, and the slow light phenomenon weakens. The reason is that the tunneling between cavity modes leads to the rapid phase dispersion in the hybrid cavity optomechanical system, which affects the group delay. Therefore, we can change the group delay $\tau$ by adjusting the tunneling strength and then change the slow light effect. In addition, we also find that the group delay of the system is negative at $\delta=\omega_{m}$, which indicates that there is a fast light phenomenon in the system.

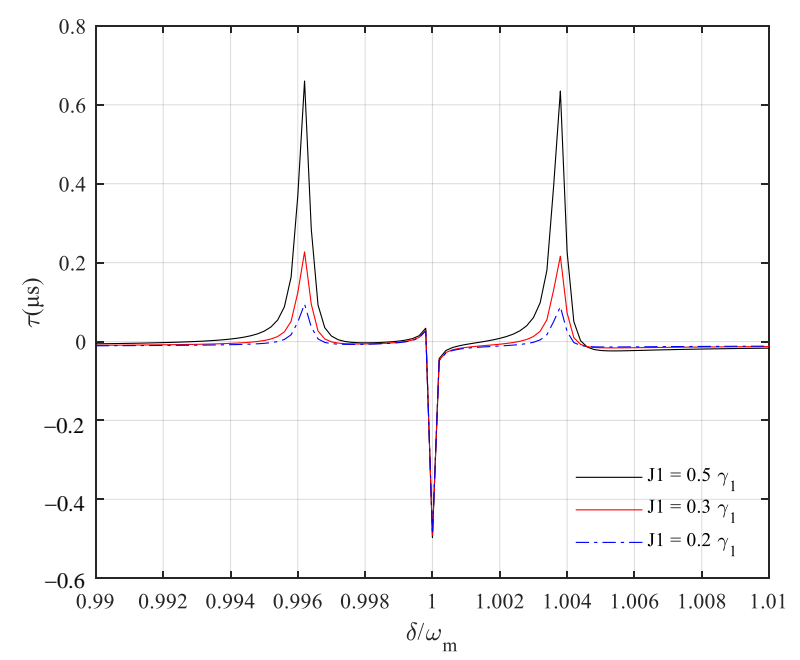

Figure 6. Group delay $\tau$ as a function of the probe detuning $\delta / \omega_{m}$ for different values of tunneling strength $J_{1}$. $J_{1}=0.5 \gamma_{1}$ (black, solid curve); $J_{1}=0.3 \gamma_{1}$ (red, dashed curve); $J_{1}=0.2 \gamma_{1}$ (blue, dash-dotted curve). $\chi=5 \times 10^{-9} \gamma_{1}$, and the other parameter values are the same as in Figure 5 .

Finally, we discuss the influence of the change in the driving field strength on the group delay. According to Figure 7, the group delay of the optomechanical system is positive near the points $\delta=0.996 \omega_{m}$ and $\delta=1.004 \omega_{m}$, and the slow light phenomenon will appear. When the strength of the driving field increases, the group delay of the system near the points $\delta=0.996 \omega_{m}$ and $\delta=1.004 \omega_{m}$ is almost unchanged, which shows that the slow light phenomenon will not change with the change of the driving field. Meanwhile, it also means that the group delay at the two points is robust to the driving field. In addition, the group delay is negative at $\delta=\omega_{m}$, which indicates that the slow light effect appears in the system. Furthermore, we can note that the depth of the group delay decrease in Figure 7 increases significantly with the increase in driving strength, and the fast light phenomenon is more obvious. The reason is that the frequency of the driving field acting on the passive cavity satisfies the boundary transition condition, so the anti-Stokes scattering appears. Moreover, the formed anti-Stokes photons have quantum interference with the photons in the passive cavity, which makes the optomechanically induced transparency appear. The appearance of the optomechanically induced transparency makes the dispersion of the system change rapidly, and the group delay changes. Therefore, we can change the fast light phenomenon by adjusting the driving field strength. 


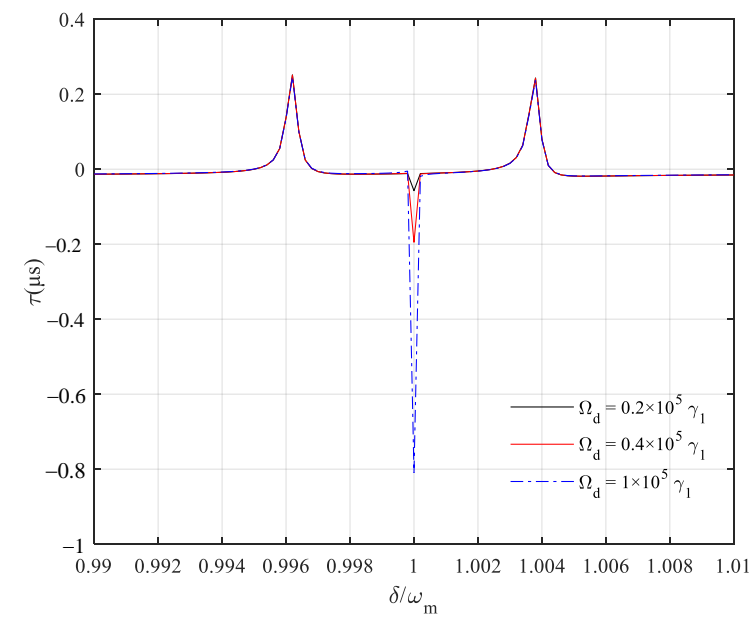

Figure 7. Group delay $\tau$ as a function of the probe detuning $\delta / \omega_{m}$ for different values of driving strength $\Omega_{d}$. $\Omega_{d}=0.2 \times 10^{5} \gamma_{1}$ (black, solid curve); $\Omega_{d}=0.4 \times 10^{5} \gamma_{1}$ (red, dashed curve); $\Omega_{d}=1 \times 10^{5} \gamma_{1}$ (blue, dash-dotted curve), $\chi=5 \times 10^{-9} \gamma_{1}$, and the other parameter values are the same as in Figure 5.

\section{Conclusions}

In summary, we study the optical response properties and the fast and slow light effects in a four-mode optomechanical system with the Kerr medium. Firstly, we study the dynamic characteristics of the hybrid cavity optomechanical system by the Langevin equation and obtain the Jacobian matrix. According to the matrix, the Lyapunov exponent is obtained, and the stability of the system is studied. It is found that when the Kerr coefficient $\chi$ changes, the stability of the optomechanical system will also change. Additionally, the optical response of the output field is obtained by the Langevin equation, and the OMIT phenomenon in the hybrid cavity optomechanical system is studied. It is found that when the optomechanical system is in a stable state, the existence of the Kerr medium can significantly enhance the absorption at $\delta=\omega_{m}$. In addition, when the value of the Kerr coefficient is increased, the left absorption peak of the system moves to the left, and the moving distance is linear with the value of the Kerr coefficient. It provides a new idea for the measurement of the Kerr coefficient. We also study the influence of the parameters in the output field on the fast and slow light effects. We have confirmed that the fast and slow light effects can be controlled by changing the Kerr coefficient, tunneling strength and driving field strength. This study provides new tools for controlling and engineering light propagation, and has potential applications in designing quantum optical devices.

Author Contributions: Conceptualization, Q.L.; Methodology, Q.L. and W.N.; Software, W.N. and X.X.; Validation, Y.L.; Formal analysis, W.B.; Investigation, Q.L., W.B. and X.X.; Resources, Q.L.; Writing-original draft preparation, W.B.; writing-review and editing, Q.L. and Y.L.; Supervision, Q.L. and Y.L.; Project administration, Q.L.; funding acquisition, Q.L. and Y.L. All authors have read and agreed to the published version of the manuscript.

Funding: This project was supported by the National Natural Science Foundation of China (Grant Nos. 62061028, 11674390, 91736106, 91836302), the Foundation for Distinguished Young Scientists of Jiangxi Province (Grant No. 20162BCB23009), the Open Research Fund Program of the State Key Laboratory of Low-Dimensional Quantum Physics (Grant No. KF202010), the Interdisciplinary Innovation Fund of Nanchang University (Grant No. 9166-27060003-YB12), and the Open Research Fund Program of Key Laboratory of Opto-Electronic Information Acquisition and Manipulation of Ministry of Education (Grant No. OEIAM202004).

Institutional Review Board Statement: Not applicable.

Informed Consent Statement: Not applicable. 
Data Availability Statement: No new data were created or analyzed in this study. Data sharing is not applicable to this article.

Conflicts of Interest: The authors declare no conflict of interest.

\section{References}

1. Aspelmeyer, M.; Kippenberg, T.J.; Marquardt, F. Cavity optomechanics. Rev. Mod. Phys. 2013, 86, 1391-1452. [CrossRef]

2. Marquardt, F.; Chen, J.P.; Clerk, A.A.; Girvin, S.M. Quantum theory of cavity-assisted sideband cooling of mechanical motion. Phys. Rev. Lett. 2007, 99, 093902. [CrossRef] [PubMed]

3. Wilson-Rae, I.; Nooshi, N.; Zwerger, W.; Kippenberg, T.J. Theory of ground state cooling of a mechanical oscillator using dynamical backaction. Phys. Rev. Lett. 2007, 99, 093901. [CrossRef]

4. He, B.; Yang, L.; Lin, Q.; Xiao, M. Radiation pressure cooling as a quantum dynamical process. Phys. Rev. Lett. 2017, 118, 233604. [CrossRef] [PubMed]

5. Lau, H.K.; Clerk, A.A. Ground-state cooling and high-fidelity quantum transduction via parametrically driven bad-cavity optomechanics. Phys. Rev. Lett. 2020, 124, 103602. [CrossRef]

6. Vitali, D.; Gigan, S.; Ferreira, A.; Böhm, H.R.; Tombesi, P.; Guerreiro, A.; Vedral, V.; Zeilinger, A.; Aspelmeyer, M. Optomechanical entanglement between a movable mirror and a cavity field. Phys. Rev. Lett. 2007, 98, 030405. [CrossRef]

7. Yan, X.B.; Deng, Z.J.; Tian, X.D.; Wu, J.H. Entanglement optimization of filtered output fields in cavity optomechanics. Opt. Express 2019, 27, 24393-24402. [CrossRef]

8. Rabl, P. Photon blockade effect in optomechanical systems. Phys. Rev. Lett. 2011, 107, 063601. [CrossRef]

9. Liao, J.Q.; Nori, F. Photon blockade in quadratically coupled optomechanical systems. Phys. Rev. A 2013, 88, 023853. [CrossRef]

10. Xu, X.W.; Li, Y.J.; Liu, Y.X. Photon-induced tunneling in optomechanical systems. Phys. Rev. A 2013, 87, 025803. [CrossRef]

11. Wang, D.Y.; Bai, C.H.; Liu, S.T.; Wang, H.F. Distinguishing photon blockade in a PT-symmetric optomechanical system. Phys. Rev. A 2019, 99, 043818. [CrossRef]

12. Børkje, K. Critical quantum fluctuations and photon antibunching in optomechanical systems with large single-photon cooperativity. Phys. Rev. A 2020, 101, 053833. [CrossRef]

13. Wang, D.Y.; Bai, C.H.; Liu, S.T.; Zhang, S.; Wang, H.F. Photon blockade in a double-cavity optomechanical system with nonreciprocal coupling. New J. Phys. 2020, 22, 093006. [CrossRef]

14. Liu, J.S.; Yang, J.Y.; Liu, H.Y.; Zhu, A.D. Photon blockade by enhancing coupling via a nonlinear medium. Opt. Express 2020, 28, 18397-18406. [CrossRef] [PubMed]

15. Wang, D.Y.; Bai, C.H.; Xing, Y.; Liu, S.T.; Zhang, S.; Wang, H.F. Enhanced photon blockade via driving a trapped $\Lambda$-type atom in a hybrid optomechanical system. Phys. Rev. A 2020, 102, 043705. [CrossRef]

16. Zhu, J.; Ozdemir, S.K.; Xiao, Y.F.; Li, L.; He, L.; Chen, D.R.; Yang, L. On-chip single nanoparticle detection and sizing by mode splitting in an ultrahigh-Q microresonator. Nat. Photon. 2010, 4, 46-49. [CrossRef]

17. Li, J.J.; Zhu, K.D. All-optical mass sensing with coupled mechanical resonator systems. Phys. Rep. 2013, 525, 223-254. [CrossRef]

18. Liu, F.; Alaie, S.; Leseman, Z.C.; Hossein-Zadeh, M. Sub-pg mass sensing and measurement with an optomechanical oscillator. Opt. Express 2013, 21, 19555-19567. [CrossRef]

19. Jing, H.; Özdemir, S.K.; Lü, X.Y.; Zhang, J.; Yang, L.; Nori, F. PT-symmetric phonon laser. Phys. Rev. Lett. 2014, 113, 053604. [CrossRef]

20. Jing, H.; Özdemir, S.K.; Geng, Z.; Zhang, J.; Lü, X.Y.; Peng, B.; Yang, L.; Nori, F. Optomechanically-induced transparency in parity-time-symmetric microresonators. Sci. Rep. 2015, 5, 9663. [CrossRef]

21. Zhang, X.Y.; Zhou, Y.H.; Guo, Y.Q.; Yi, X.X. Double optomechanically induced transparency and absorption in parity-timesymmetric optomechanical systems. Phys. Rev. A 2018, 98, 033832. [CrossRef]

22. Li, W.L.; Jiang, Y.F.; Li, C.; Song, H.S. Parity-time-symmetry enhanced optomechanically-induced-transparency. Sci. Rep. 2016, 6, 31095. [CrossRef] [PubMed]

23. Zhang, X.Y.; Guo, Y.Q.; Pei, P.; Yi, X.X. Optomechanically induced absorption in parity-time-symmetric optomechanical systems. Phys. Rev. A 2017, 95, 063825. [CrossRef]

24. Xiao, X.; Liao, Q.H.; Zhou, N.R.; Liu, Y.C. Tunable optical second-order sideband effects in a parity-time symmetric optomechanical system. Sci. China-Phys. Mech. Astron. 2020, 63, 1-8.

25. Yan, X.B. Optomechanically induced transparency and gain. Phys. Rev. A 2020, 101, 043820. [CrossRef]

26. Manipatruni, S.; Robinson, J.T.; Lipson, M. Optical nonreciprocity in optomechanical structures. Phys. Rev. Lett. 2009, 102, 213903. [CrossRef] [PubMed]

27. Qu, K.; Agarwal, G.S. Fano resonances and their control in optomechanics. Phys. Rev. A 2013, 87, 063813. [CrossRef]

28. Ruesink, F.; Mathew, J.P.; Miri, M.A.; Alù, A.; Verhagen, E. Optical circulation in a multimode optomechanical resonator. Nat. Commun. 2018, 9, 1-6. [CrossRef]

29. Lai, D.G.; Wang, X.; Qin, W.; Hou, B.P.; Nori, F.; Liao, J.Q. Tunable optomechanically induced transparency by controlling the dark-mode effect. Phys. Rev. A 2020, 102, 023707. [CrossRef]

30. Chang, D.E.; Safavi-Naeini, A.H.; Hafezi, M.; Painter, O. Slowing and stopping light using an optomechanical crystal array. New J. Phys. 2011, 13, 023003. [CrossRef]

31. Monroe, C. Quantum information processing with atoms and photons. Nature 2002, 416, 238-246. [CrossRef] 
32. Braunstein, S.L.; Van Loock, P. Quantum information with continuous variables. Rev. Mod. Phys. 2005, 77, 513. [CrossRef]

33. Agarwal, G.S.; Huang, S. Electromagnetically induced transparency in mechanical effects of light. Phys. Rev. A 2010, 81, 041803. [CrossRef]

34. Huang, S.; Agarwal, G.S. Electromagnetically induced transparency from two-phonon processes in quadratically coupled membranes. Phys. Rev. A 2011, 83, 023823. [CrossRef]

35. Weis, S.; Rivière, R.; Deléglise, S.; Gavartin, E.; Arcizet, O.; Schliesser, A.; Kippenberg, T.J. Optomechanically induced transparency. Science 2010, 330, 1520-1523. [CrossRef] [PubMed]

36. Safavi-Naeini, A.H.; Alegre, T.P.M.; Chan, J.; Eichenfield, M.; Winger, M.; Lin, Q.; Hill, J.T.; Chang, D.E.; Painter, O. Electromagnetically induced transparency and slow light with optomechanics. Nature 2011, 472, 69-73. [CrossRef] [PubMed]

37. Li, X.Y.; Nie, W.J.; Chen, A.X.; Lan, Y.H. Effect of the mechanical oscillator on the optical-response properties of an optical trimer system. Phys. Rev. A 2018, 98, 053848. [CrossRef]

38. Peng, J.X.; Chen, Z.; Yuan, Q.Z.; Feng, X.L. Optomechanically induced transparency in a Laguerre-Gaussian rotational-cavity system and its application to the detection of orbital angular momentum of light fields. Phys. Rev. A 2019, 99, 043817. [CrossRef]

39. Han, Y.; Cheng, J.; Zhou, L. Electromagnetically induced transparency in a cavity optomechanical system with an atomic medium. J. Phys. B At. Mol. Opt. Phys. 2011, 44, 165505. [CrossRef]

40. Gu, K.H.; Yan, D.; Wang, X.; Zhang, M.L.; Yin, J.Z. Hybrid electromagnetically-optomechanically induced transparency in an atom-assisted optomechanical system. J. Phys. B At. Mol. Opt. Phys. 2019, 52, 105502. [CrossRef]

41. Han, C.M.; Wang, X.; Chen, H.; Li, H.R. Tunable slow and fast light in an atom-assisted optomechanical system with a mechanical pump. Opt. Commun. 2020, 456, 124605. [CrossRef]

42. Liao, Q.H.; Xiao, X.; Nie, W.J.; Zhou, N.R. Transparency and tunable slow-fast light in a hybrid cavity optomechanical system. Opt. Express 2020, 28, 5288-5305. [CrossRef]

43. Liu, J.H.; Yu, Y.F.; Zhang, Z.M. Nonreciprocal transmission and fast-slow light effects in a cavity optomechanical system. Opt. Express 2019, 27, 15382-15390. [CrossRef]

44. Boyd, R.W.; Gauthier, D.J. Controlling the velocity of light pulses. Science 2009, 326, 1074-1077. [CrossRef]

45. Jiao, Y.; Lü, H.; Qian, J.; Li, Y.; Jing, H. Nonlinear optomechanics with gain and loss: Amplifying higher-order sideband and group delay. New J. Phys. 2016, 18, 083034. [CrossRef]

46. Jiao, Y.F.; Lu, T.X.; Jing, H. Optomechanical second-order sidebands and group delays in a Kerr resonator. Phys. Rev. A 2018, 97, 013843. [CrossRef]

47. Lü, H.; Jiang, Y.J.; Wang, Y.Z.; Jing, H. Optomechanically induced transparency in a spinning resonator. Photonics Res. 2017, 5, 367-371. [CrossRef]

48. Zimmer, F.; Fleischhauer, M. Sagnac interferometry based on ultraslow polaritons in cold atomic vapors. Phys. Rev. Lett. 2004, 92, 253201. [CrossRef]

49. Shahriar, M.S.; Pati, G.S.; Tripathi, R.; Gopal, V.; Messall, M.; Salit, K. Ultrahigh enhancement in absolute and relative rotation sensing using fast and slow light. Phys. Rev. A 2007, 75, 053807. [CrossRef]

50. Wang, B.; Liu, Z.X.; Kong, C.; Xiong, H.; Wu, Y. Mechanical exceptional-point-induced transparency and slow light. Opt. Express 2019, 27, 8069-8080. [CrossRef]

51. Ziauddin; Rahmatullah; Hussain, A.; Abbas, M. Double transparency with slow and fast light in an optomechanical system. Opt. Commun. 2020, 461, 125284. [CrossRef]

52. Kumar, T.; Bhattacherjee, A.B.; Mohan, M. Dynamics of a movable micromirror in a nonlinear optical cavity. Phys. Rev. A 2010, 81, 013835. [CrossRef]

53. Huang, S.; Agarwal, G.S. Enhancement of cavity cooling of a micromechanical mirror using parametric interactions. Phys. Rev. A 2009, 79, 013821. [CrossRef]

54. Farman, F.; Bahrampour, A.R. Effects of optical parametric amplifier pump phase noise on the cooling of optomechanical resonators. J. Opt. Soc. Am. B 2013, 30, 1898-1904. [CrossRef]

55. Shahidani, S.; Naderi, M.H.; Soltanolkotabi, M.; Barzanjeh, S. Steady-state entanglement, cooling, and tristability in a nonlinear optomechanical cavity. J. Opt. Soc. Am. B 2014, 31, 1087-1095. [CrossRef]

56. Li, L.; Nie, W.J.; Chen, A.X. Transparency and tunable slow and fast light in a nonlinear optomechanical cavity. Sci. Rep. 2016, 6, 35090. [CrossRef]

57. Shahidani, S.; Naderi, M.H.; Soltanolkotabi, M. Control and manipulation of electromagnetically induced transparency in a nonlinear optomechanical system with two movable mirrors. Phys. Rev. A 2013, 88, 053813. [CrossRef]

58. Genes, C.; Vitali, D.; Tombesi, P.; Gigan, S.; Aspelmeyer, M. Ground-state cooling of a micromechanical oscillator: Comparing cold damping and cavity-assisted cooling schemes. Phys. Rev. A 2008, 77, 033804. [CrossRef]

59. Giovannetti, V.; Vitali, D. Phase-noise measurement in a cavity with a movable mirror undergoing quantum Brownian motion. Phys. Rev. A 2001, 63, 023812. [CrossRef]

60. Xiong, H.; Si, L.G.; Zheng, A.S.; Yang, X.X.; Wu, Y. Higher-order sidebands in optomechanically induced transparency. Phys. Rev. A 2012, 86, 013815. [CrossRef]

61. Liu, Z.H.; Lai, Y.C.; Matías, M.A. Universal scaling of Lyapunov exponents in coupled chaotic oscillators. Phys. Rev. E 2003, 67, 045203. [CrossRef] [PubMed] 
62. DeJesus, E.X.; Kaufman, C. Routh-Hurwitz criterion in the examination of eigenvalues of a system of nonlinear ordinary differential equations. Phys. Rev. A 1987, 35, 5288-5290. [CrossRef]

63. Zhang, J.Q.; Li, Y.; Feng, M.; Xu, Y. Precision measurement of electrical charge with optomechanically induced transparency. Phys. Rev. A 2012, 86, 053806. [CrossRef]

64. Massel, F.; Heikkilä, T.T.; Pirkkalainen, J.M.; Cho, S.U.; Saloniemi, H.; Hakonen, P.J.; Sillanpää, M.A. Microwave amplification with nanomechanical resonators. Nature 2011, 480, 351-354. [CrossRef] [PubMed]

65. Zhao, X.H.; Hou, B.P.; Liu, L.; Zhao, Y.H.; Zhao, M.M. Cross-Kerr effect in a parity-time symmetric optomechanical system. Opt. Express 2018, 26, 18043-18054. [CrossRef] [PubMed] 